Prepared for the U.S. Department of Energy

under Contract DE-AC05-76RL01830

\title{
Outcomes of the 2013 GTO Workshop on Geothermal Code Comparison
}

TD Scheibe

MD White

SK White

March 2013






\title{
DISCLAIMER
}

This report was prepared as an account of work sponsored by an agency of the United States Government. Neither the United States Government nor any agency thereof, nor Battelle Memorial Institute, nor any of their employees, makes any warranty, express or implied, or assumes any legal liability or responsibility for the accuracy, completeness, or usefulness of any information, apparatus, product, or process disclosed, or represents that its use would not infringe privately owned rights. Reference herein to any specific commercial product, process, or service by trade name, trademark, manufacturer, or otherwise does not necessarily constitute or imply its endorsement, recommendation, or favoring by the United States Government or any agency thereof, or Battelle Memorial Institute. The views and opinions of authors expressed herein do not necessarily state or reflect those of the United States Government or any agency thereof.

\author{
PACIFIC NORTHWEST NATIONAL LABORATORY \\ operated by \\ BATTELLE \\ for the \\ UNITED STATES DEPARTMENT OF ENERGY \\ under Contract DE-AC05-76RL01830
}

Printed in the United States of America
Available to DOE and DOE contractors from the Office of Scientific and Technical Information,
P.O. Box 62, Oak Ridge, TN 37831-0062;
ph: (865) 576-8401
fax: $(865)$ 576-5728
email: reports@adonis.osti.gov

\begin{abstract}
Available to the public from the National Technical Information Service, U.S. Department of Commerce, 5285 Port Royal Rd., Springfield, VA 22161 ph: (800) 553-6847 fax: $(703) 605-6900$ email: orders@ntis.fedworld.gov online ordering: http://www.ntis.gov/ordering.htm
\end{abstract}






\section{Introduction}

Pacific Northwest National Laboratory (PNNL) is supporting the Department of Energy (DOE) Geothermal Technologies Office (GTO) in organizing and executing a model comparison activity. This project is directed at testing, diagnosing differences, and demonstrating modeling capabilities of a worldwide collection of numerical simulators for evaluating geothermal technologies.

Numerical simulation codes increasingly have become critical tools for understanding complex processes in geologic media, and currently are being applied to assess technology feasibilities, design geologic systems, evaluate field observations, and guide operational procedures. The increased use of numerical simulation for geologic systems comes from 1) continued validation of numerical simulators against laboratory experiments and field observations, yielding growing confidence in numerical simulation; 2) a need for evaluating coupled processes in geologic systems, driven by geologic sequestration of greenhouse gases and unconventional energy sources; and 3) advances in computing technologies, enabling increased model complexity and grid resolution. To become a trusted analytical tool for geothermal technologies numerical simulation codes must be tested to demonstrate that they adequately represent the hydrologic, thermodynamic, geochemical, and geomechanical processes of concern.

The principal issues of concern for this project are to determine 1) do we have valid mathematical models for the fundamental processes associated with geothermal technologies?; and 2) can available numerical simulators assimilate these models to yield reliable and accurate numerical solutions to problems involving conditions of practical interest? The intent for this project is that participants with available numerical simulators will benefit from testing and comparing their codes, diagnosing differences with other codes, and identify needs in simulation capabilities and additional research.

The project is divided into three distinct stages: 1) organization, 2) execution, 3) documentation. During the organization stage participants will be solicited, problem coordinators identified, problem sets developed, and participation protocols established. During the execution stage participating teams will apply their codes to the selected problems and submit simulation results, and the project coordinators will develop comparisons of the model outcomes; some iteration is likely as modeling teams learn about the problems and solutions and refine their approaches. We are applying a web-based knowledge and data management framework for numerical simulation and modeling known as Velo ${ }^{1}$, developed at the Pacific Northwest National Laboratory, during both the organization and execution stages of the project and as a portal for the dynamic code comparison archive. The final stage will be documentation of the results, which will include development of project reports and journal manuscripts for publication in the open scientific literature.

A key element of the organization stage, currently underway, was the planning and implementation of a one-day project kickoff workshop, held February 14, 2013 in Palo Alto, CA. The primary goals of the workshop were to 1) introduce the project and its objectives to potential participating team members, and 2) develop an initial set of test problem descriptions for use in the execution stage. The workshop was timed to immediately follow the Stanford University Geothermal Workshop (held Feb 12-13), so as to maximize participation and minimize travel costs and schedule intrusions for participants. This report summarizes the outcomes of the Feb. 14 GTO workshop.

\footnotetext{
${ }^{1}$ Gorton, I., C. Sivaramakrishnan, G. Black, S. White, S. Purohit, M. Madison, K. Schuchardt. 2011. "Velo: Riding the knowledge management wave for simulation and modeling." In Proceedings of 4th International Workshop on Software Engineering for Computational Science and Engineering, SE-CSE 11, May 28, 2011, Waikiki, Honolulu, HI, USA.
} 


\section{Participants}

Forty-three participants registered for the workshop, including the workshop organizers. The participant list is provided below (workshop organizers are denoted with an asterisk). Participants represented a range of organizations including academic institutions, national laboratories, industry, and international entities.

\begin{tabular}{|c|c|c|}
\hline Last Name & First Name & Institution \\
\hline Anderson & Brian & West Virginia University \\
\hline Baer & Kristian & Technische Universitaet Darmstadt \\
\hline Bakane & Piyush & University of Nevada, Reno \\
\hline Blankenship & Douglas & Sandia National Laboratories \\
\hline *Boyd & Lauren & U.S. Department of Energy \\
\hline Cladouhos & Trenton & AltaRock Energy \\
\hline Danko & George & Univ. of Nevada, Reno \\
\hline Ezzedine & Souheil & LLNL \\
\hline Fairhurst & Charles & Itasca Consulting Group, Inc. \\
\hline Finsterle & Stefan & LBNL \\
\hline $\mathrm{Fu}$ & Pengcheng & Lawrence Livermore National Laboratory \\
\hline Garcia & Julio & Calpine - The Geysers \\
\hline Ghassemi & Ahmad & University of Oklahoma \\
\hline Hickman & Stephen & USGS \\
\hline Hoang & Viet & Chevron Energy Technology Co. \\
\hline Horne & Roland & Stanford University \\
\hline Huang & Hai & INL \\
\hline Juliusson & Egill & Landsvirkjun, Iceland \\
\hline Kelkar & Sharad & LANL \\
\hline Kennedy & Mack & Lawrence Berkeley National Laboratory \\
\hline McClure & Mark & University of Texas at Austin \\
\hline Pettitt & Will & Itasca Consulting Group, Inc. \\
\hline Petty & Susan & AltaRock Energy \\
\hline *Phillips & Benjamin & U.S. Department of Energy \\
\hline Plummer & Mitch & Idaho National Laboratory \\
\hline *Podgorney & Robert & Idaho National Laboratory \\
\hline Polsky & Yarom & ORNL \\
\hline Riahi & Azadeh & Itasca Consulting Group, Inc. \\
\hline Roegiers & Jean-Claude & University of Oklahoma \\
\hline Ruhaak & Wolfram & Technische Universitaet Darmstadt \\
\hline Rutqvist & Jonny & Lawrence Berkeley National Laboratory \\
\hline *Scheibe & Tim & Pacific Northwest National Laboratory \\
\hline Smith & Katherine (Kat) & Idaho National Laboratory \\
\hline Sonnenthal & Eric & Lawrence Berkeley National Lab \\
\hline Stillman & Greg & U.S. Department of Energy \\
\hline
\end{tabular}




$\begin{array}{lll}\text { Taron } & \text { Joshua } & \text { USGS } \\ \text { Uddenberg } & \text { Matt } & \text { Altarock Energy } \\ \text { Vandermeer } & \text { William } & \text { Department of Energy } \\ \text { Wang } & \text { Herb } & \text { University of Wisconsin-Madison } \\ * \text { White } & \text { Mark } & \text { Pacific Northwest National Laboratory } \\ * \text { White } & \text { Signe } & \text { Pacific Northwest National Laboratory } \\ \text { Wu } & \text { Yu-Shu } & \text { Colorado School of Mines } \\ \text { Ziagos } & \text { John P } & \text { LLNL }\end{array}$

\section{Venue}

The workshop was held on the Stanford University campus, at the Li Ka Shing center for Learning and Knowledge. Stanford Professor Roland Horne served as our campus sponsor and was in attendance at the workshop.
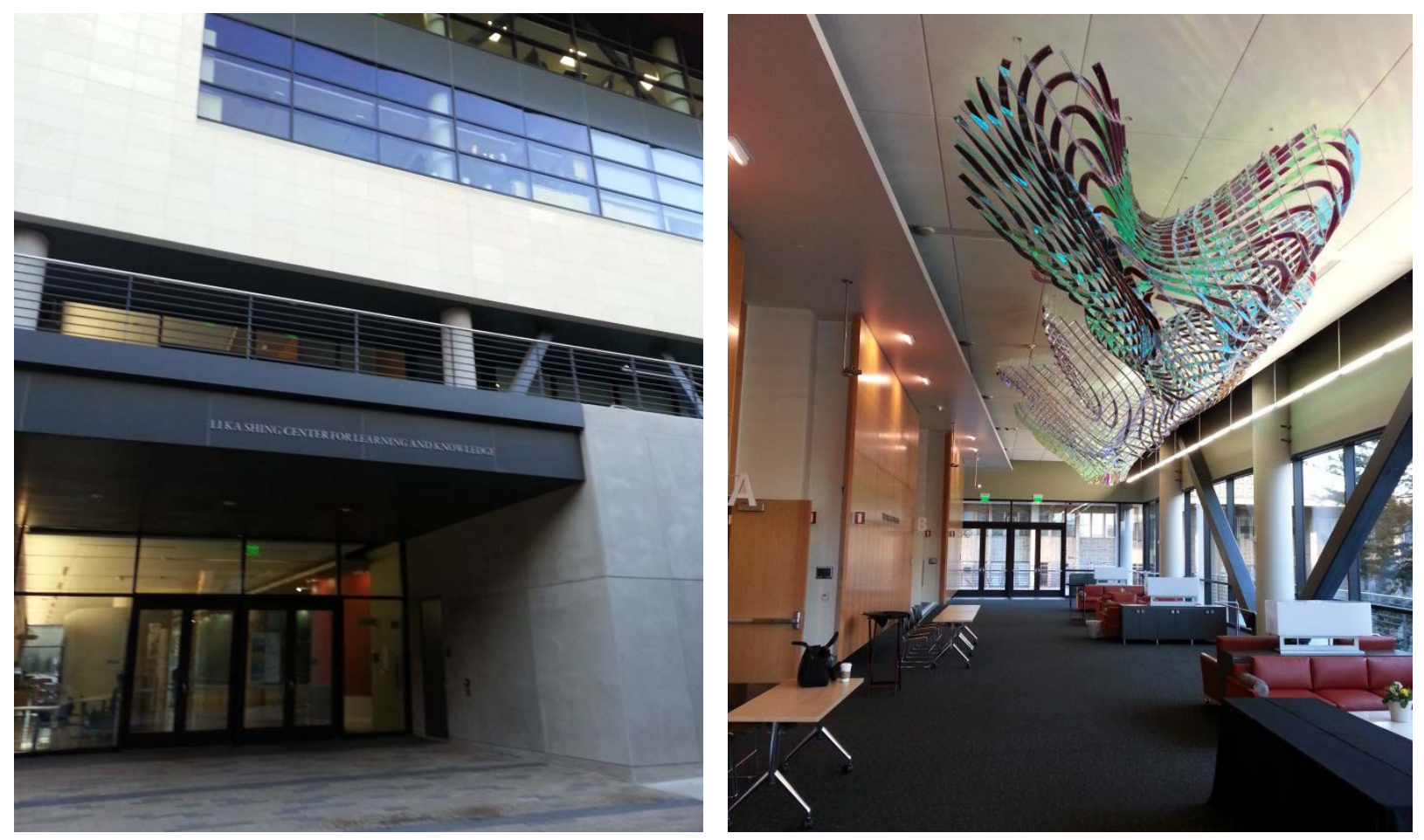


\section{Agenda}

The meeting agenda is provided here. A full day of activities was planned, with presentations and discussion in the morning and breakout sessions in the afternoon.

7:45 AM Breakfast on your own

8:15 AM Welcome and Introductions Tim Scheibe / Lauren Boyd

9:00 AM Summary of IPGT Activities and Status Rob Podgorney

10:00 AM Break

10:15 AM Demonstration of Velo

11:00 AM Example Problem Presentation

11:30 AM Discussion

Noon Lunch on your own

1:00 PM Breakout Session \#1

1A: Benchmark Problems

1B: Test Cases

Facilitator: Signe White

Facilitator: Tim Scheibe

1C: Challenge Problems

Facilitator: Rob Podgorney

(Floating Facilitators: Mark White / Lauren Boyd / Ben Phillips)

2:30 PM Break (Switch Breakout Sessions)

2:40 PM Breakout Session \#2

2A: Benchmark Problems

2B: Test Cases

2C: Challenge Problems

(Floating Facilitators: Mark White / Lauren Boyd / Ben Phillips)

3:35 PM Break (Facilitators/Scribes Compile Breakout Session Results)

4:00 PM Breakout Facilitators Report Back (15 min each)

4:45 PM Discussion and Next Steps

Facilitator: Tim Scheibe

Facilitator: Signe White

Facilitator: Tim Scheibe

Facilitator: Rob Podgorney

\section{5:30 PM Meeting Adjourns}




\section{Morning Presentations}

Lauren Boyd (DOE), Acting Supervisor for the GTO Enhanced Geothermal Systems (EGS) Program Area, provided the opening remarks and welcomed the workshop participants. Lauren presented the Program's goals and objectives for the code comparison study and for the workshop. Following Lauren's presentation, each participant briefly introduced him/herself so that all participants could gain awareness of the breadth of the group's background and represented organizations.

Rob Podgorney (Idaho National Laboratory) provided a presentation of the International Partnership for Geothermal Technology (IPGT) working group on Geothermal Modeling. The IPGT recently held its own workshop (September 2012 in Switzerland) to initiate an international code-comparison effort. The DOE project is being closely coordinated with the international effort, and IPGT member country representatives were invited to this workshop. The Velo web-based knowledge and data management framework is being used collaboratively by both organizations, and information on test problems and model solutions will be extensively shared between the two efforts to maximize benefit. Rob presented the initial suite of problems that were defined by the IPGT working group, as a potential starting point for discussion regarding selection of problems for the DOE study. The IPGT defined three categories of test problems: 1) Benchmarks (simple problems for which analytical solutions are known); 2) Test Cases (more complex but well-constrained problems for which there may be no analytical solution but for which all codes are expected to provide

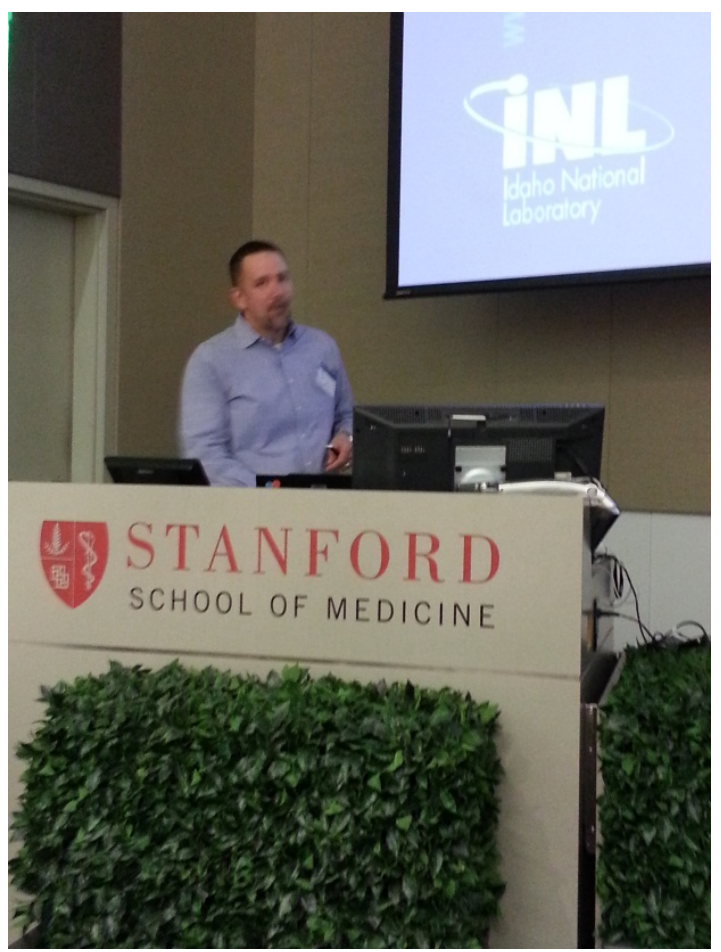
comparable solutions, and 3) Challenge Problems (highly complex problems with extensive process coupling, perhaps based on field experimental data, for which the solution is not known a priori and different codes may be expected to provide different solutions). We used this framework to organize our breakout sessions (see Agenda above). Following Rob's presentation there was substantial discussion regarding the objectives of the DOE code comparison effort and its relationship to the IPGT effort. Key comments or questions that came up in the discussion included:

- Does this study include seismic wave process modeling?

- It was generally agreed that the IPGT problem set included too many "benchmark" problems and that our focus should be more narrowly on problems specifically related to EGS modeling.

- It was suggested to develop a database describing the functionality and methods embodied in the various codes (Note: This comment is currently being addressed within Velo development efforts).

- Caution is needed for complex problems with many parameters - such a model can fit anything! Sensitivity analysis may help to identify which parameters are most important.

- Understanding of process models and how they are implemented is important, but must be framed within the context of real geologic systems.

- There were several comments regarding the potential use of field data, and how that might influence the direction of this study.

- How are participants in this effort going to be funded? 
Signe White (Pacific Northwest National Laboratory) provided a short demonstration to workshop attendees of the Velo framework as currently deployed on an externally accessible server at PNNL. Those who had wireless access and had previously requested a Velo account were able to follow along on their own laptops. The Velo system is a wiki-based tool for information management and collaboration that has been customized for the GTO study. Key functional elements for early stages of the GTO project include an interactive user discussion forum, information on the participating teams, an interactive database with descriptors of the functional elements of various geothermal simulation codes, sharing of relevant documents and indexing using defined keywords, and a variety of administrative and security functions. Most important for this project are pages defining the test problems and team areas to which problem solutions can be uploaded. Because the wiki platform allows user interaction (content editing), these pages are not static but rather can be developed and added to by the user community. The image below shows a screen capture of the Velo tool, with a page in view that describes one of the IPGT Test Cases.

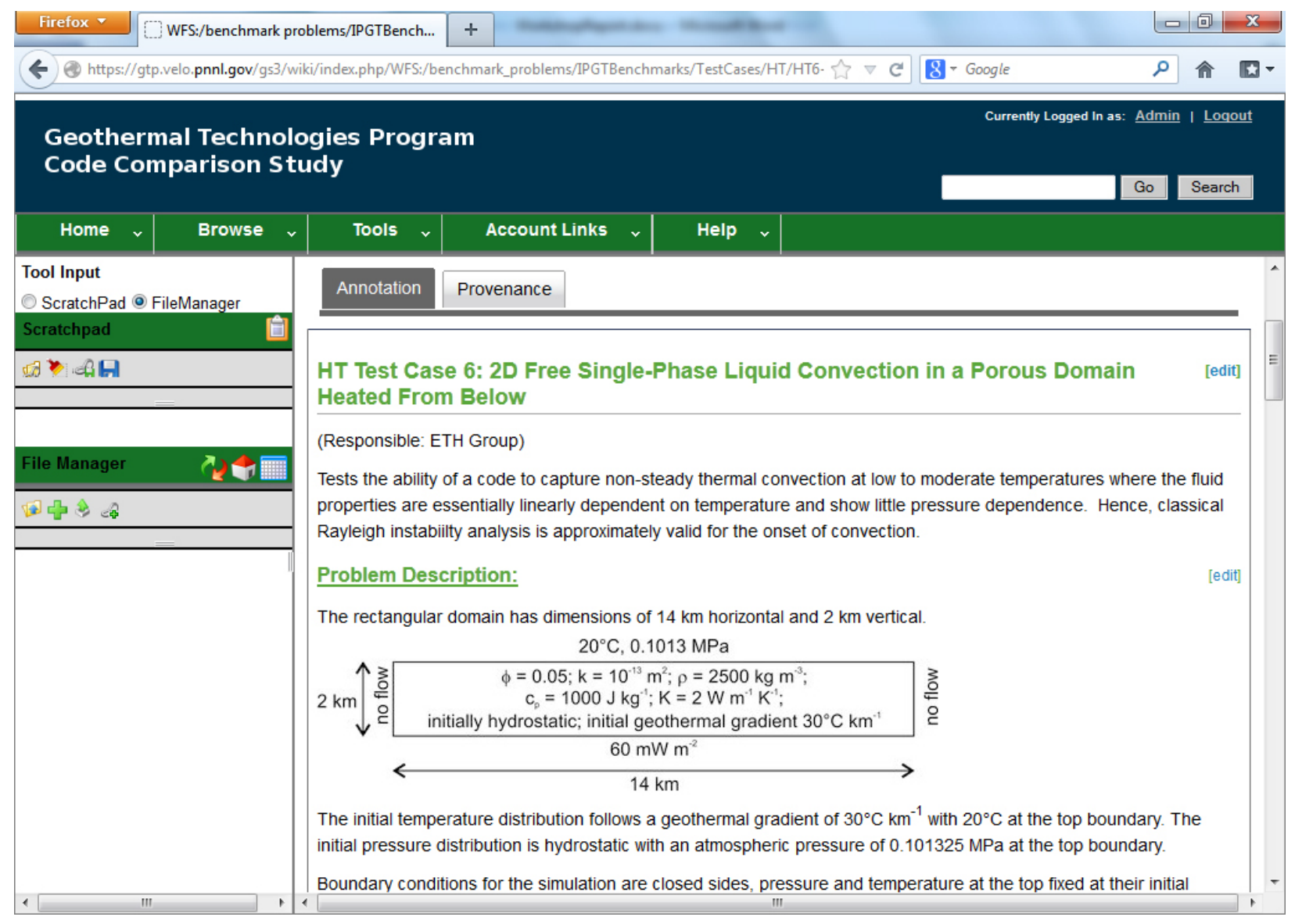

As part of the Velo demonstration, Mark White presented a sample problem with several solutions developed using different modeling assumptions (see Figure below). This problem was designed to illustrate aspects of good problem design such as clear definition of underlying assumptions, specification of all necessary inputs, and definition of a problem with a well-constrained solution. 

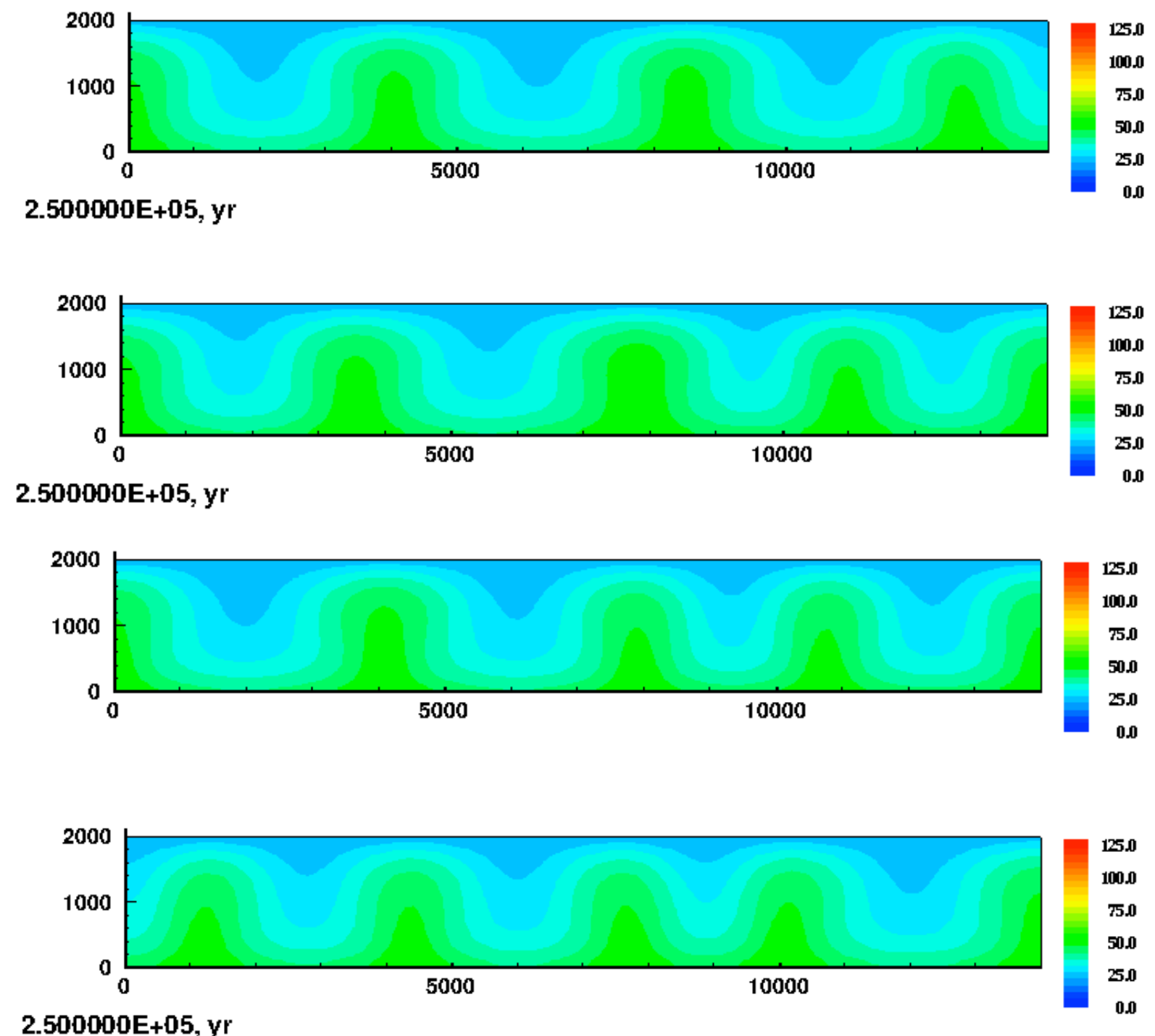

\section{$2.500000 \mathrm{E}+05, \mathrm{yr}$}

Figure: Four different solutions to a sample Test Case problem (IPGT Test Case HT\#6), presented as part of the Velo demonstration. In each simulation, slight differences in model assumptions were used. The outcomes (all plotted at the same simulated time) show temperature profiles in a density-instable system with variable numbers of plumes predicted. top) Base scenario; top center) modified initial condition (assumed constant density for vertical pressure gradient rather than hydrostatic with variable density); bottom center) decreased grid spacing from $100 \mathrm{~m}$ to $50 \mathrm{~m}$; bottom) removed 100 year limit on maximum time step.

Following the Velo demonstration, Tim Scheibe (Pacific Northwest National Laboratory) went over the plans for the breakout sessions and encouraged all participants to sign up for a breakout session in each of the two afternoon time slots. A brief discussion time was held, in which it was emphasized to the workshop participants that the IPGT problem set was considered only a starting point, and that it is important for this group to develop their own set of problems well suited to our specific objectives. Following this discussion, the group adjourned for lunch on our own. 


\section{Breakout Sessions}

The afternoon part of the workshop focused on two consecutive breakout sessions. Originally it was planned to have three parallel breakout sessions in each time period as shown in the agenda above, but based on the morning discussion and the distribution of breakout signups we decided to combine the Benchmarks and Tests Cases into a single breakout, with the second breakout focused on Challenge Problems. By running each breakout session twice, we allowed each workshop participant to contribute to each of the two topics. Tim Scheibe and Signe White facilitated the Benchmarks/Tests Cases sessions, and Rob Podgorney facilitated the Challenge Problems sessions. Other workshop organizers moved between the different sessions and took notes. Prior to the workshop a set of questions was defined for the breakout sessions to guide the discussion and provide an outline for note taking. These questions are listed below:

1. Critical EGS Issues:

What code capabilities for EGS simulation are currently most limiting their application?

$\checkmark$ What are the attributes of codes for EGS simulation that are most important to evaluate/test/compare?

2. How well does the IPGT suite of problems cover the necessary scope?

Is there consensus on the general approach? Are any modifications to the framework needed?

Are any of the problems unnecessary?

Are any problems missing?

3. What Metrics Are Most Useful for Comparison of Model Outputs?

This is clearly problem-specific to some extent, but see if some general themes can be identified. Example: Time-histories of a monitored state variable at selected points in the domain at a specified time interval.

4. Logistical Issues:

File formats (primarily output) and interpolation (grid type and resolution, who is responsible?)

$\checkmark$ Computational limitations

$\checkmark$ Proprietary information

Other:

5. Use of Velo:

Any concerns, issues or questions?

Ideas for suggested enhancements or custom tools?

6. Feedback on Template for Problem Description:

Suggestions for improved structure?

$\checkmark$ Necessary information that is missing?

Most of the discussion time was focused on the definition of problems (question set 2 above). During each breakout session there were assigned scribes that took notes using the provided template, and notes were also recorded on flip charts in the rooms. Following the breakout sessions the notes were quickly compiled by the facilitators into a provided PowerPoint template and used to give an oral summary report to the full group at the conclusion of the workshop. Key points from each of the two breakout session topics are summarized below. 


\section{Breakout Session Summary: Benchmarks/Test Cases}

The breakout sessions on Benchmarks and Test Cases were very productive in that a number of specific problems were outlined that will form a strong basis for initial code comparison efforts. The responses of the participants to each question set are summarized (from both sessions) below:

Question set 1: What code capabilities for EGS simulation are currently most limiting their application? What are the attributes of codes for EGS simulation that are most important to evaluate/test/compare?

$\checkmark$ Complex geometry / grids

$\checkmark \quad$ Fracture propagation in 3D (or even 2D)

$\checkmark$ Reservoir scale vs. wellbore scale

$\checkmark$ Uncertainty evaluation

$\checkmark$ Process coupling

$\checkmark$ Computational limitations

$\checkmark$ Insufficient validation, lack of confidence

$\checkmark$ Different fluid types (equations of state, e.g. water, $\mathrm{CO}_{2}$ )

Question set 2: How well does the IPGT suite of problems cover the necessary scope? Is there consensus on the general approach? Are any modifications to the framework needed? Are any of the problems unnecessary? Are any problems missing?

$\checkmark$ There was general agreement on the framework adopted by the IPGT, although everyone agreed that we should reduce the number of simple benchmarks and focus on problems relevant to EGS. We focused this discussion on development of test problems, with the assumption that a sufficient set of benchmarks is being defined by the IPGT and we can selectively solve those as needed.

$\checkmark$ Nine potential test case problems were put forward by the workshop participants. For each of these, a schematic diagram of the problem was presented and specific elements of the proposed problem were discussed. Sufficient information was provided such that the project leads can put initial problem descriptions up on Velo and the participants can subsequently collaboratively fill in the details. The nine problems proposed are briefly described below. The alphabetic designations indicate the classes of processes incorporated in the problem ( $\mathrm{T}=$ thermal; $\mathrm{H}=$ hydraulic; $\mathrm{M}=$ mechanical; $\mathrm{C}=$ chemical).

1. Poroelastic / thermal transport in a single fracture (THM): This problem involves a single pre-existing planar fracture in hot rock with initially uniform aperture. Cold fluid is injected at a specified rate at one end of the fracture and transported to the other end. The model must account for poroelastic effects due to increased pressure and thermal cooling effects, which will lead to changes in the fracture aperture, and must predict the temperature profile over time of the effluent fluid.

2. Shear on a single fracture induced by change in effective stress by injection (HM/THM): In this problem, a single fracture or joint exists in a 3D hot rock domain at some specified distance from an injection well. Fluid is injected at the well, and due to either or both temperature and/or pressure effects the normal stress on the existing fracture is reduced, leading to hydroshearing.

3. Calcite dissolution/precipitation in a fracture/pipe (THC): This problem will have a similar physical configuration as problem \#1, but will be in a calcium carbonate rock that interacts 
chemically with the injected fluid. The model must simulate the dissolution and precipitation profile along the length of the fracture using a prescribed reaction network including both equilibrium and kinetic reactions.

4. Calculation of stress field incipient to fracturing and comparison to laboratory data (HM): The person who proposed this problem is performing laboratory experiments in which solid rock columns with geometric irregularities are subjected to external stress and the stress field within the rock (including around the tip of the irregularity) is directly measured. The model must incorporate the specified system geometry and compute the stress field as the external stress is increased. The model should predict the onset of fracturing when the stress at the tip of the irregularity exceeds the incipient fracturing stress value.

5. IPGT Test Case THM \#4: The group liked the IPGT's Test Case THM \#4, and suggested that it be applied to a deterministically-specified fracture network system. This test case involves thermal and possibly hydraulic stimulation over a multi-week period, and tests the ability to simulate mechanical response (including potentially shearing and fracture growth) and the resultant change in permeability and production. The IPGT problem will be based on data collected in conjunction with the EGS Demonstration at the Raft River site.

6. Inverse modeling: It was suggested that at least one problem should have an element of parameter estimation. Suggested problem type is a discrete fracture network simulated as an effective continuum. For this problem, limited data (system responses) would be provided in the problem description and the model would be required to estimate other system properties (known only to the problem designers) using inverse modeling methods. It was agreed that there should be relatively few degrees of freedom; too much complexity would lead to difficulty in interpreting results. Optimally the methods used would also consider uncertainty both in inferred model parameters and simulated outcomes of some specified prediction scenario.

7. Propagation of an open fracture from a well bore (HM/THM): Simulation of a pre-existing closed fracture or joint with residual aperture that resolves into a hydraulic fracture over time due to pressure stimulation at a well.

8. Surface deformation from a pressurized fracture (HM): In this problem (which could possibly be an extension or variant of problem \#7), the model would be required to predict surface deformations associated with pressurization of a fracture.

9. Disc or lens-shaped existing fracture around a borehole with porous media beyond (THM): In this problem, fluids are circulated within a pre-existing fracture between injection and production points (along the lines of Fenton Hill site or huff/puff testing in Germany). Thermal drawdown cannot be explained using a static fracture geometry; the model must account for mechanical effects and stress-dependent permeability changes.

Question set 3: What metrics are most useful for comparison of model outputs?

$\checkmark$ Need to specify consistent units or nondimensional variables

$\checkmark$ Important for this to be clearly defined in the problem description

$\checkmark$ Whether or not UQ/sensitivity is assessed is contingent on the problem description 
Question set 4: Logistical issues

$\checkmark \quad$ The primary outcome of this discussion was identification of major file formats used by participant codes. Most codes provide output in various ASCII text formats; several also use VTK format and one uses Exodus. For time series data, comma-separated values (CSV) format is common.

Question set 5: Use of Velo

$\checkmark \quad$ It was suggested that summary instructions on how to use the Velo system be provided to users as an introduction (probably after the problem sets are better defined and some tools are added)

$\checkmark$ Tools are needed for visualization of multiple problem solutions (can probably use Velo tools built around VTK/ParaView currently under development)

Question set 6: Feedback on problem description template (Appendix A)

$\checkmark$ Detailed descriptions of input grid and time discretizations are needed - a "suggested" grid should be provided but users can choose to do something different

$\checkmark$ Specify constitutive relationships used or assumed (e.g., viscosity as f(temperature))

\section{Breakout Session Summary: Challenge Problems}

The breakout sessions on Challenge Problems led to extensive discussion regarding the objectives of the study and in particular the relative merits of using synthetic problem descriptions versus data from an EGS field site as the basis for challenge problem development. In a synthetic problem, the focus would be on testing the representation of physics and the methods of numerical simulation against a fully-known and well-constrained system (i.e., focus on the codes). In contrast, a field-based problem would test the physic representation and numerical solution, but would also incorporate differences in model conceptualization (i.e., tests both the code and the conceptual model approach). Notes taken on a flip chart in the room regarding this discussion (critical aspects of each approach) are tabulated below:

Challenge Problem Scope Discussion

\begin{tabular}{|l|l|}
\hline (TEST PHYSICS) - Code only & $\begin{array}{l}\text { (TEST PHYSICS AND APPROACH) - Code } \\
\text { and conceptual model }\end{array}$ \\
\hline Accessible & Aspirational \\
\hline Lab or Field scale? & Field-based \\
\hline $\begin{array}{l}\text { Synthetic system (or blend - can incorporate } \\
\text { selected field data) }\end{array}$ & $\begin{array}{l}\text { Comprehensive / diverse data (chemistry, } \\
\text { microseismicity, etc.) }\end{array}$ \\
\hline Agree on key processes to capture & Close collaboration with operator \\
\hline Key observables that constrain & Choose a demo site? \\
\hline Well-constrained approach by all teams & Modeling team picks approach (conceptualization) \\
\hline Logistically practical? & Integrate with upcoming operations? \\
\hline
\end{tabular}

Because these discussions were more philosophical in nature, the groups did not get to the point of specifying actual problem types or initial descriptions. In fact, it was decided that it would be better to start with the Test Cases to develop the process for code comparison and collaboration, with subsequent 
definition of Challenge Problems to be based on lessons learned from the Test Cases. Key points of the discussion are summarized below, in the context of the six question sets.

Question set 1: What code capabilities for EGS simulation are currently most limiting their application?

$\checkmark$ The assumption that fracture slips are going to cause MEQS can be limiting in less seismic or aseismic environments

$\checkmark$ Have to assume parameters of the stress field at some sites

$\checkmark$ Field data almost always yields some mysteries

$\checkmark$ We don't understand the relationship between new and existing fractures and how they interact during hydroshearing

$\checkmark$ Reservoir behavior can switch from porous to fracture dominated as flow rates increase

$\checkmark$ We need more straightforward input parameters

What are the attributes of codes for EGS simulation that are most important to evaluate/test/compare?

$\checkmark$ Models that can handle real world geologic heterogeneity and anisotropy

$\checkmark$ Basic physics of shear enhanced permeability, how tensile cracks propagate

$\checkmark$ Lithology / stress state

$\checkmark$ Simulate specific conditions of fracture intersections / sensitivity analysis

$\checkmark$ How to handle bulk permeability issues in continuum versus discrete fracture models?

$\checkmark$ Where is the continuum approach appropriate, and where is DFN approach more appropriate?

$\checkmark$ How to handle scale effects? How to handle localized flow?

$\checkmark$ The more that can be predicted about observable values from the field, the better and the easier it is to tune (less black box effect)

$\checkmark$ Data assimilation for the purposes of model updates is optimal. Fitting pressure, temperature, and flux data is optimal (versus fitting just one set)

$\checkmark$ Start with a simple model, then add intricacies as required

Question set 2: How well does the IPGT suite of problems cover the necessary scope? Is there consensus on the general approach? Are any modifications to the framework needed? Are any of the problems unnecessary? Are any problems missing?

$\checkmark$ Instead of having so many problems to choose from, pick the one problem with the most robust data set

o Possibilities: Fenton Hill lots of existing injection, temp, flow data; Soultz?

o What is the minimum general criteria that would be required for sites: seismic arrays, tracers, pressure and temperature data?

o Fundamental Processes: shear induced fractures

o Observables in the Field: thermal profiles, tracer data,

$\checkmark$ Should we use a site with more complex or less complex geology?

$\checkmark$ Do we want to look at one site or multiple sites? Single well versus multi well sites? Greenfield versus operating field?

$\checkmark$ Do we need to characterize an economically successful EGS site?

$\checkmark$ Seems to be a preference for historical projects with more data availability versus newer projects that have yet to stimulate 
$\checkmark$ Use a developmental approach (much like Phases I, II, and III of actual EGS Demonstration Project) on a real field site as it would be in the real world where more and more input data is added with time

$\checkmark$ test cases can include inverse study

$\checkmark$ Question from group: What is the overall goal, correctness of model predictions versus highlighting differences between codes? Are we challenging the modeler or challenging the code, principally? A synthetic problem could provide a better control

$\checkmark$ Probabilistic results could be more useful that deterministic results

$\checkmark$ Should none of the models predict some of the actual field data, that could be indicative of a physics issue

$\checkmark \quad$ Try to explain more difficult problems (seismicity at Newberry and tracer returns at Desert Peak)?

$\checkmark$ Should we be picking the failure mode (hydroshear versus hydrofrac) on the front end?

$\checkmark$ Oil and gas purposefully use competing models when analyzing potential effects from proposed well stimulation plans

$\checkmark$ Borrow test parameters (stress states, etc.) from real world cases (e.g., Soultz), then add boundaries as necessary

$\checkmark \quad$ The IPGT problem set does not including any problems that include prediction of a microseismicity plot from stimulation activities; needs to be solved probabilistically

Question set 3: What metrics are most useful for comparison of model outputs?

$\checkmark$ Sensitivity Study from each team with a model would be good (i.e. what inputs have the most impact on the output?) temperature/seismicity/strain/etc. A simple, synthetic type of problem could be used here.

$\checkmark$ Spatial information on temperature, Pressure, total power/energy output, changes in stress and strain across the reservoir, microseismicity, surface deformation

$\checkmark$ Assessment of uncertainty, non-uniqueness, etc. regarding results

$\checkmark$ Fractured Volume, Distribution, Density

$\checkmark$ Fracture Surface Area

$\checkmark$ Could create a flowchart for industry to use for development of EGS projects based on modeling predictions

$\checkmark$ Could run a session on modeling results to be presented at GRC (for example)

$\checkmark$ General message from panel: Need to define more specific objectives for the code comparison exercise prior to commencement

Question set 4: Logistical issues

$\checkmark$ Similar results as in the other breakout session

Question set 5: Use of Velo

$\checkmark$ Comparison tools for temperature outputs from observed data would be useful

$\checkmark$ Suggested to use Velo as a group forum to review problem definitions

Question set 6: Feedback on problem description template (Appendix A)

$\checkmark \quad$ Need to provide place for data sets from field site(s) that are utilized 


\section{Next Steps}

The workshop organizers held a follow-up conference call during the week following the workshop to review the workshop outcomes and define steps forward. It was recognized that there were two major questions that came up repeatedly during the workshop, for which we need to formulate a consistent response.

1. What is the overarching objective of the project, and how does that impact the design of the problems? What do we hope to learn, and what are the boundaries? Do we intend to limit the scope to code comparison, or do we endeavor to undertake a full-fledged model comparison (including effects of conceptual model and data interpretation by different modeling groups)?

2. Should the challenge problems focus on synthetic systems or real field data?

While these two questions continue to be debated, our current path forward assumes that our goal is to provide a platform for understanding the inherent assumptions and different approaches of various codes, which can only be rigorously observed when a common problem is solved under controlled and constrained conditions. It is not necessary at this point to fully define the challenge problems; we should undertake work on the several test case problems that were identified and use the results of those efforts to help define issues to be addressed by one or more challenge problems. For the challenge problems, it may be best to start with a fully constrained synthetic problem (for example, following some of the concepts used by the petroleum community in the SPE benchmark efforts), and subsequently move to a more field-based problem that incorporates modeling choices as well as code differences.

The critical next step during the next month is to develop initial problem definitions for the nine test cases outlined above, put those descriptions on Velo, and initiate the community input process. Currently there

are over twenty external users that have set up Velo accounts, and it is important to get them involved and using the Velo interface as soon as possible. It is also important to make concrete steps toward defining the composition of participating teams - who will be involved, what codes they will be using, and how they will be funded? 


\section{Appendix A: Problem Description Template}

O PROBLEM TITLE

O (Responsible: [Name(s)])

o Problem Description:

o [Introductory Text Here (one paragraph)]

o System Configuration:

o [Describe system geometry, dimensionality, processes incorporated, etc. Include graphics as necessary]

o Initial and Boundary Conditions:

o [Specify initial and boundary conditions. Include graphics as necessary.]

o Property Table:

o [TABULATE THREE COLUMNS: 1) Property Name, 2) Units, 3) Value(s) or reference to file]

o Metrics

o [Define the model outputs to be used for comparing solutions. Are there any standards for comparison, expected results, etc.]

0 References

o [Provide literature citations as appropriate (and upload documents to file manager if available)]

o Example Solution:

o Disclaimer

$0 \quad[$ Text here as needed]

o Methods

o [Describe the methods used to solve the problem (code used, grid resolution, other modeling decisions.]

o Supporting Files

o [Describe supporting files that are provided (i.e., input and output files). Files should be uploaded to the File Manager.]

o Results

o [Describe results for the example solution. Provide graphs and visualization plots as needed.] 